\title{
Get to know your ABC Editors - a virtual interview
}

\author{
Nicola Oberbeckmann-Winter ${ }^{1}[$
}

Accepted: 8 November 2021 / Published online: 15 December 2021

(c) Springer-Verlag GmbH Germany, part of Springer Nature 2021

Analytical and Bioanalytical Chemistry (ABC) is the society journal for rapid publication and global visibility of (bio-) analytical research - but who are the Editors of $\mathrm{ABC}$ ? The team currently consists of 11 internationally renowned analytical scientists:

Antje J. Baeumner (University of Regensburg, Germany), Hua Cui (University of Science and Technology of China, Hefei, China), Günter Gauglitz (University of Tübingen, Germany), Gérard Hopfgartner (University of Geneva, Switzerland), Luigi Mondello (University of Messina, Italy), María C. Moreno-Bondi (Complutense University of Madrid, Spain), Sabine Szunerits (University of Lille, France), Qiuquan Wang (Xiamen University, China), Stephen A. Wise (National Institutes of Health, Bethesda, USA), Joseph Zaia (Boston University, USA) and Chair Editor Adam T. Woolley (Brigham Young University, Provo, USA).

Learn how they feel about being an ABC Editor, advice they give to authors and readers, and other insights they are willing to share with you.

\section{What do you like most about being an $A B C$ Editor?}

Luigi Mondello: From a young age, I have been strongly motivated to learn about chemistry. Being an Editor of ABC gives me the opportunity to follow new trends and be constantly updated in the field of analytical (and bioanalytical) chemistry.

Published in the topical collection celebrating ABCs 20th Anniversary.

Nicola Oberbeckmann-Winter

nicola.oberbeckmann-winter@springer.com

1 Analytical and Bioanalytical Chemistry, Springer, Tiergartenstrasse 17, 69121 Heidelberg, Germany
Qiuquan Wang: The thing I like most about being an Editor of $\mathrm{ABC}$ is that $\mathrm{I}$ have an opportunity to select high-quality papers for $\mathrm{ABC}$ readers.

Stephen Wise: Being part of starting, building, and growing $\mathrm{ABC}$ from its beginning has been an exciting, challenging, satisfying, and occasionally frustrating experience. As an Editor of $\mathrm{ABC}$ for the past 20 years, the most enjoyable aspect has been the association with the other editors and the $\mathrm{ABC}$ editorial staff. It has been a pleasure to interact with these exceptional editors in making $\mathrm{ABC}$ a premier journal in analytical and bioanalytical chemistry. During my professional career, I have always enjoyed preparing scientific research papers. Serving as an ABC Editor has provided a unique opportunity to collaborate with authors, and particularly with my own research colleagues, to encourage and promote the preparation and publication of quality manuscripts.

Adam Woolley: I love working with my editor and publishing colleagues in charting a course toward a bright and promising future for $\mathrm{ABC}$.

Joseph Zaia: I enjoy facilitating the development of emerging bioanalytical fields by publishing exciting manuscripts from leading research groups from around the world.

\section{What advice would you give to a first-time ABC author?}

Antje Baeumner: Read ABC papers and find those that tell a great scientific story; those that best present their data; and those that spark your interest just by reading title, abstract and possibly conclusions. Now, try to apply those strategies to writing your own manuscript. After you have finished writing it, let it be for a week without thinking about it. Then, look at it again as if you were an interested reader. Your data are likely presented in the best format already, but does your abstract really portray your scientific idea, findings and excitement? If yes, you are ready to go and submit it. If no, change it until it excites you again. 
Sabine Szunerits: Being an editor is a great responsibility as the editor is the first person to have access to new research data and findings with the responsibility to evaluate and decide if the paper is of the standard for the journal, $\mathrm{ABC}$ in this case. The multidisciplinary character of the sensing field often makes it challenging. What to do with such papers? ABC is focusing on the analytical chemistry and sensing community. It is thus essential for a manuscript to have a clear analytical aspect with analytical parameters (e.g., limit of detection, sensitivity, selectivity). If these remain marginal, even if other aspects are original, it is not in the scope of $\mathrm{ABC}$ and needs to be revised or even rejected.

Qiuquan Wang: Write and submit a concise and innovative academic paper. It is the most important thing for you to know what you want to tell ABC readers. Why did you do the study? How did you do it? What achievements did you make? Is there any novelty in instrumentation and/or method development as well as application compared with the already existing ones? Did you solve a problem that the available technologies and methods are not able to do?

\section{What advice would you give to someone the first time they have a proposal or paper rejected?}

Antje Baeumner: Don't worry, this happens to everyone and happens to the best among us. After you have digested your disappointment, read the reviewers' comments again and triage them in a way that helps you improve your manuscript (or proposal). For example, look for those that are actually constructive and provide you with suggestions for improvement. This is the best free advice you can get; soak it up and learn. Sometimes, it may only be different ways of presenting data or your story and highlight aspects you may not have included before. Sometimes it seems as if reviewers didn't understand what you were trying to say. Take it as advice that you were not clear enough in your description (and your initial reflex response to suggest that the reviewers are simply not smart or had no time to read your manuscript carefully may not necessarily be the right response). In the end, keep in mind, reviewers are just like you; they are your peers, from whom you have the privilege, through our peer-review system, to learn. Finally, after you have improved your great manuscript (or proposal) carefully, resubmit it and you will likely succeed. Günter Gauglitz: Carefully read the reviewers' comments, consider them, reconsider your work and resubmit.

María Moreno-Bondi: First of all, and especially at the beginning of your career, one should not think that a rejected paper is the end of the world, just a stone on the road. Do not be discouraged by it. I suggest that you make a careful evaluation of the reasons for the rejection of your paper. Rejection letters often include reviewers' comments that can help the authors identify the errors detected in the manuscript. In fact, a rejection does not mean that the entire work should be discarded. They should analyse the reasons for rejection, as objectively as possible, and draw conclusions that will allow them to plan the necessary activities to improve the quality of the work before a new submission. Authors should always keep in mind the aims and scope of the target journal and check that the content of their paper is within that scope, as this is an important cause of paper rejections.

Adam Woolley: All professionals in the field of analytical and bioanalytical chemistry deal with the disappointment of rejection. When that happens, it helps to take a deep breath, focus on the larger view, learn from the feedback given, and move forward, which sometimes means proceeding in a different direction.

\section{What is the impact of $A B C$ on the analytical community?}

Antje Baeumner: ABC's papers go a step beyond most analytical journals and present the combination of new principles/techniques/materials/strategies in combination with real-world samples. Thus, with an ABC paper, you'll know that the new knowledge described has a fair chance to truly provide new solutions to existing challenges. Günter Gauglitz: ABC supplies facts instead of fake news and quantitative data to many daily problems, as outlined in various $\mathrm{ABC}$ editorials, such as the one on the social impact of analytical chemistry.

Qiuquan Wang: Actually, $\mathrm{ABC}$ has a long history. It evolved from Fresenius' Journal of Analytical Chemistry and others about 20 years ago. $\mathrm{ABC}$ is one of the most influential academic journals in the field of analytical and bioanalytical chemistry.

\section{What is the most important lesson you have learned over your career?}

Antje Baeumner: If you add these three aspects to your own modus operandi portfolio - resilience, collaboration, mentoring - you will always be able to succeed.

Hua Cui: You should develop a long-term research vision, and keep working on it in a systematic manner, regardless of how hot the research field is at a given point.

Gérard Hopfgartner: As the world is changing rapidly, knowledge and experience does not make you smarter to manage a career, just wiser to get around difficult situations and to appreciate good ones.

Luigi Mondello: The most important lesson I have learned in my career is that it is only with enthusiasm and hard work that you can reach important achievements. In other words, "love what you do". If you love it, you will do it well.

María Moreno-Bondi: The most important lessons I have learned throughout my career are mainly these two: 1) to 
achieve something, we must work hard and pursue what we believe in, to the end; and 2) research work benefits from teamwork and collaborations with experts that improve the quality of the research and are very beneficial for the training of young researchers. A multidisciplinary approach is a must in science nowadays.

Sabine Szunerits: One never stops learning, but research has its up and downs. The downs (rejection of a paper, funding not accepted, student losing motivation, etc.) are generally more present in a career than the ups (paper accepted, successful grant application, student finishes their PhD, etc.). The lesson to be learned: celebrate the moment of glory and resources yourself, as hard times come sooner than one can imagine.

Qiuquan Wang: Working diligently and wisely is the most important thing.

Stephen Wise: Collaborate, collaborate, and collaborate! Early in my research career, I developed collaborations with a number of other analytical chemists that were very beneficial for advancing our shared research interests. These collaborators in academia, government, and industry were also early in their careers as analytical chemists, and we were excited to share our research with each other and to utilize our complementary strengths and resources (usually analytical techniques) to produce better research outcomes and ultimately better publications. Multiple visits to each other's laboratories over the years were essential in these collaborations. The immediate outcome from these partnerships were excellent publications in various analytical journals. However, the enduring benefit has been the personal friendships that were developed. Today, I consider these collaborators not only as professional colleagues but as personal friends. One of these collaborators was already an established leader in analytical chemistry when we met, and fortunately for me, he welcomed our collaboration and became a valuable mentor for me. In fact, he was responsible for introducing me to Fresenius' Journal of Analytical Chemistry, the major forerunner of $\mathrm{ABC}$, which commenced my involvement with that journal and ultimately led to my appointment as one of the founding Editors of $\mathrm{ABC}$. My advice is to seek out opportunities to collaborate with other analytical scientists and to develop these personal relationships. Joseph Zaia: Science is an emotional endeavor. One wants to make a contribution to one's field. One wants to have one's contributions recognized. But it is best not to get caught up in one's emotions, even when one experiences a setback.

\section{Have you ever encountered 'serendipity' in your scientific endeavors?}

Hua Cui: When we prepared hydrogels consisting of chitosan, $\mathrm{CL}$ reagent $N$-(4-aminobutyl)- $N$-ethylisoluminol and catalyst $\mathrm{Co}^{2+}$, we accidentally discovered that they could generate firefly-mimicking intensive and long-lasting chemiluminescence. The light emission was even visible to the naked eye and lasted for over $150 \mathrm{~h}$ when the hydrogels were mixed with $\mathrm{H}_{2} \mathrm{O}_{2}$. Further investigation demonstrated that this was attributed to slow-diffusion-controlled heterogeneous catalysis.

Gérard Hopfgartner: Serendipity plays a major role in scientific endeavors. It can come either through collaborations or incorrect experimental conditions, which enable one to get unexpected novel results or insights.

\section{What career options outside of the analytical chemistry field did you consider?}

Antje Baeumner: For the longest time I was interested in bioprocess engineering and environmental biotechnology - i.e., sustainable and clean production of complicated compounds, and cleaning up the environment through the help of microorganisms. These had outcompeted my interest in pursuing a medical degree by the time I entered college. Now I am passionate about developing (bio)sensors for clinical diagnostics, environmental and food monitoring. I guess, I didn't stray very far.

Günter Gauglitz: I was considering business administration or industrial engineering alternatively to analytical chemistry.

María Moreno-Bondi: I always enjoyed history because I think that the study of the past helps us to understand human behaviour and changes in societies. As I read once, history is "the laboratory of human experience", and I would like to learn about this different "type of lab". I don't discard the idea of studying for this degree during retirement.

Qiuquan Wang: I wanted to be a medical doctor when I was young.

\section{What is your favorite non-scientific activity?}

Hua Cui: I like traveling to discover the beauty of nature and to record it by photography.

Gérard Hopfgartner: Winter sports and in particular crosscountry skiing and of course to heat up a fondue afterwards.

Luigi Mondello: I am very busy with my academic role and scientific career but, if (when) I have time, I love to spend time by the sea (especially sailing and scuba diving) and relax at home with my family and my cats, reading comics and cooking. Sabine Szunerits: Classical music needs a conductor, first and second violins and other instruments and all harmoniously aligned to result in a nice piece of music. That is the same in research. Therefore, my favourite non-scientific activity is listening to classical music and enjoying the presence of musicians. Qiuquan Wang: Travel to see the world.

Stephen Wise: In my youth, I was a coin collector, and this hobby greatly expanded my interest in and knowledge of world geography. 
I never imagined at that time that I would eventually visit many of these countries during my career as an analytical chemist. My coincollecting hobby was dormant for nearly 40 years; however, during my professional travels, returning with current or old coins and currency from the various countries visited has been an obligation. For the past 45 years, I have assembled a significant library of books related to the history and culture of the area where I grew up. Early in my career when I would travel to a scientific meeting in various cities in the USA, I would always spend an afternoon visiting the used/rare bookstores in the city looking for exceptional bargains (and I did make a couple of nice finds!). On many of these book-hunting excursions, I would be accompanied by an analytical chemist colleague who also had an interest in books in a specific field. However, with the advent of eBay in the mid-1990s, used/rare book collecting changed drastically, and visits to bookstores became significantly less productive. My dilemma now is that I have interests in collecting too many different items, including books, coins and currency, and nineteenth century photographs and maps of the American West. Eventually, I would like to research some of these vintage photos and possibly write about them.

Adam Woolley: Family is really important to me, but since that's technically not an activity, we'll go with seasonally appropriate "i"-containing outdoor activity verbs: biking, hiking, fishing, and skiing.

Joseph Zaia: I enjoy swimming as a meditative and restorative physical activity.
Thank you to the team of ABC Editors for sharing their ideas and views!
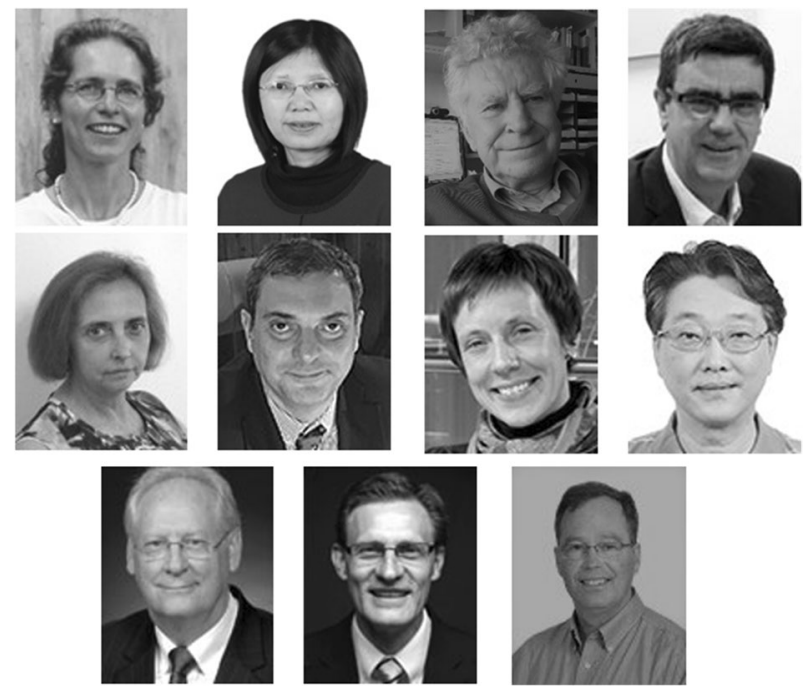

(Left to right, top to bottom) Antje Baeumner, Hua Cui, Günter Gauglitz, Gérard Hopfgartner, Maria Moreno-Bondi, Luigi Mondello, Sabine Szunerits, Qiuquan Wang, Stephen Wise, Adam Woolley, Joseph Zaia

Publisher's note Springer Nature remains neutral with regard to jurisdictional claims in published maps and institutional affiliations. 